\title{
Aspergillus fumigatus spondylodiscitis with bilateral psoas abscess in a renal transplant patient with pulmonary tuberculosis : A rare case report
}

\author{
Ravikumar TV ${ }^{1}$, Raghvendra Rao ${ }^{2}$, Amit Grover $^{3 *}$, Daksh Gadi $^{4}$ \\ ${ }^{1}$ Assistant Professor, Department of Orthopaedics, MS Ramaiah Medical College, Bangalore, Karnataka, India \\ ${ }^{2}$ Assistant Professor, MS Ramaiah Medical College, Bangalore, Karnataka, India \\ ${ }^{3}$ Resident, MS Ramaiah Medical College, Bangalore, Karnataka, India \\ ${ }^{4}$ Resident, MS Ramaiah Medical College, Bangalore, Karnataka, India \\ *Corresponding author E-mail:amitgrover88@gmail.com
}

\begin{abstract}
Background: Aspergillus spondylodiscitis is increasingly described in immune-compromised patients. Its diagnosis is challenging and its delay results in high mortality and morbidity in view of its relative infrequency and the non- specific clinical signs and symptoms. Case report:We report a case of thoracic spondylodiscitis caused by Aspergillus fumigatus in a patient of renal transplantation and with proven pulmonary tuberculosis.

Conclusion:We reiterate that transplantist and the treating physician should have high grade of suspicion for the invasive Aspergillosis even when the patient complains of vague non-specific back pain, and aggresively aim to rule out fungal infection. Early surgical debridement and antifungal therapy to be executedto prevent rapid progression of invasive aspergillosis and neurological damage.
\end{abstract}

Keywords:Aspergillus; Psoas Abscess; Spondylodiscitis; Tuberculosis.

\section{Background}

Aspergillus spondylodiscitis is increasingly described in immunecompromised patients. Invasive aspergillosis infection, particularly, that involving the central nervous system and spinal cord are accompanied withthe highest mortality rate .Its diagnosis is challenging and delay results in high mortality and morbidity in view of its relative infrequency and the non- specific clinical signs and symptoms. Fungal infections do occur commonly in renal transplant recipients especially in genitourinary system however reports describing such a condition are very few. Herein, we report a case of thoracic spondylodiscitis caused by Aspergillus fumigatus in a patient of renal transplantation, and with proven pulmonary tuberculosis.

\section{Case report}

We report a 34 year old man, who underwent living mother donor kidney transplantation two years earlier with persistent mild graft dysfunction, was admitted with back pain, general ill health and not able to ambulate. His past medical history revealed onset of progressive renal failure due to chronic glomerulonephritis since 2010 and was on heamodialysis for 2 years before renal transplantation in 2012. He was also proven positive for tuberculosis with acid fast staining of sputum in 2011 and had completed the antitubercular course for 18 months with renal dosage adjustment and was respiratory symptom free till the time of presentation. Post transplantation he suffered mild delayed graft rejection and was on immunosuppression with Tacrolimus, mycophenolatemofetil and prednisolone. He developed secondary hypertension for which he was managed with prazosin, dilitiazem and clonidine. One year Post transplantation he developed urosepsis due to E.coli, sensitive to amikacin and meropenem and managed with intravenous injection. In May 2014, he presented to emergency department with severe back ache, aggravated at night that could not be relieved by rest over previous 2 months along with tenderness around $8^{\text {th }}, 9$ th and $10^{\text {th }}$ thoracic spinous process and paraspinal region with intact neurology and added sounds on chest auscultation. His chest $\mathrm{x}$-ray revealed a right mediastinal mass compared to his previous $x$ rays which was suggestive of tuberculosis in view of history of previous pulmonary tuberculosis. His sputum for acid fast bacilli came negative. Computed and Magnetic resonance imaging of the chest and spine showed a spondylodiscitiswith right paravertebral collection tracking along the paraspinal region to the psoas area on both side and extending to the upper pole of the recipient kidney on right side measuring $10 * 6 * 4 \mathrm{~cm}$ (Figure 1). His blood examination revealed a Haemoglobin of 9.4, creatinine of 5.4 , Sodium 138 , Potassium 4.1, ESR 45 , Total counts of 7450 .He underwent CT guided aspiration, which did not disclose any organism. He was planned for retropleural evacuation and biopsy by costotransversectomy at Dorsal 9 vertebra. Amber coloured caseous material was drained from retropleural region and spinal instrumentation was not done in view of stable bone segments. Histopathology of the sample revealed Aspergillus fumigatus. He was put on Liposomal amphotericin B and itraconozole with careful monitoring of the side effects. 


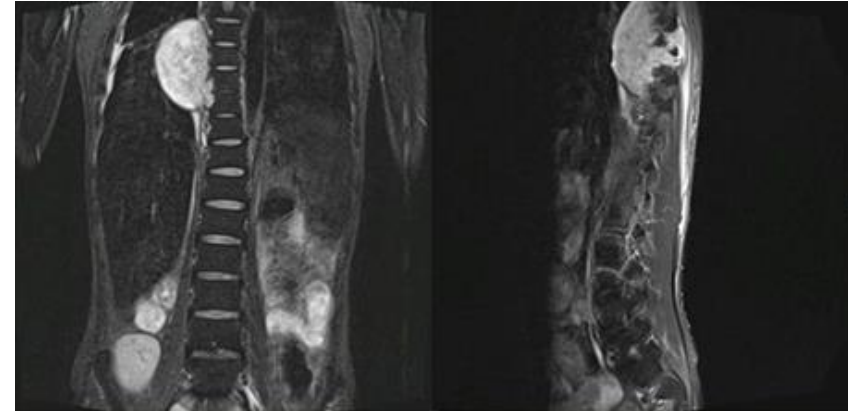

Fig. 1:MRI Showing Spondylodiscitis with Paravertebral Collection

On Post op day 14 he developed breathlessness, hypotension and with metabolic acidosis and was monitored in ICU. Next day he complained of sudden onset of weakness of upper and lower limbs predominant in lower limbs with urinary retention and his Magnetic resonance imaging of the brain and Spine revealed multiple lacunar infarcts in the frontal and parietal region with spinal cord indentation with granulation tissue from the paraspinal abscess. On post op day 16, he had deteriorating sensorium and hypotension, inspite of ionotropic support along with and he succumbed to cardiac arrest which did not respond to best efforts of CPR.

\section{Conclusion}

Case reports of Aspergillus spondylodiscitis after solid organ and multivisceral transplants have been reported (Castelli C et al.1990, Hummel M et al.1993, Park SB et al.2004, Salvalaggio PR et al.2003, Tang TJ et al. 2000, Wéclawiak H et al.2007). Multiple factors like renal failure, steroids, immunosuppression, neutropenia, defective phagocytes, bacterial and cytomegalovirus infections, long term use of IV cannulas and antibiotics, presence of vascular complications, graft rejection, diabetes, malignancy, parenteral nutrition, and dialysis increases the risk of invasive Aspergillosis. In our case the patient was predisposed to Aspergillus by a combination of multiple infections, use of immunosuppressive agents and previous use of intravenous broad-spectrum antibiotics. In our case, to facilitate the diagnosis of invasive fungal infection ion the first setting was masked by the history of proven pulmonary tuberculosis. There are very few cases of Aspergillous spondylodiscitis along with tuberculosis published in the literature (Ersoy A ET al.2007, Park SB et al.2004). We reiterate that transplantist and the treating physician should have high grade of suspicion for the invasive Aspergillosis even when the patient complains of vague non-specific back pain and aggresively aim to rule out fungal infection using imaging studies, biopsy and non-culture based diagnostic techniques such as galactomannan or DNA detection in blood and bronchoalveolar lavage sample. Early surgical debridement and antifungal therapy to be executed to prevent rapid progression of invasive aspergillosis and neurological damage (Gerlach UA etal.2008. Salvalaggio PR etal.2003).

The most important step in invasive fungal infections is to identify high-risk patients -which can reduces mortality. Acute myelogenous leukaemia,myelodysplastic syndrome, haematopoietic stem cell transplantation, recipients of solid organ transplants, severe and prolonged immunosuppression are risk factors for the invasive fungal infection(Ersoy A et al.2007).

Extensive research has been conducted to facilitate the best possible treatment strategies for these invasive fungal infections. Timing and drug of choice is controversial (Rüping MJ et al.2008).

Nonspecific back and neck pain, insidious onset and progressively worsening over several weeks or months without clinical characteristics is the primary complaint of vertebral osteomyelitis presentation. Clinically, spondylodicitis is suspected based on the clinical suspicion and radiological findings of bone destruction, paradiscal erosion and paravertebral collection along with soft tissue extension and edema. The definitive diagnosis of Aspergillus infection is revealed by demonstration of characteristic, acute branching, broad, septate hyphae in biopsy materials, and culture and also based on non-culture diagnostic techniques like galactomannan or DNA detection in blood or bronchoalveolar lavage samples

After having reviewed the major clinical trials, Ruping MJ et al (Rüping MJ et al.2008), recommended some guide lines like, a) prophylaxis with posaconazole for allogeneic haematopoietic stem cell transplantation recipients, patients receiving induction chemotherapy for Acute myelogenous leukaemia, myelodysplastic syndrome, and those undergoing immunosuppressive therapy for graft-versus-host disease after allogeneic haematopoietic stem cell transplantation, b) for the empirical treatment of persistently febrile neutropenia, caspofungin is our first- and liposomal amphotericin B deoxycholate as second-line choice, c) Once a diagnosis of invasive aspergillosis has been established, voriconazole should be the preferred treatment option, with liposomal amphotericin B deoxycholate being an alternative. Once a diagnosis has been established, the drug of choice for adequate treatment depends largely on neutrophil count and haemodynamic stability. In nonneutropenic patients, an echinocandin should be considered the first-line treatment option. In neutropenic patients, caspofungin or micafungin might be preferred to anidulafungin as first-line treatment. Liposomal amphotericin B deoxycholate is a second-line treatment option in both settings (Rüping MJET al.2008).

Early cautious treatment initiation in invasive Aspergillosis infection has impact on mortality rates; however specific diagnostic measures are lacking (Maschmeyer GET al.2007). This scenario leads to the parallel use of prophylactic, empirical and pre-emptive anti-fungal regimen treatment. Also on definite diagnosis of fungal infections targeted treatment is employed (Rüping MJet al.2008).

\section{References}

[1] Castelli C, Benazzo F, Minoli L, Marone P, Seghezzi R, Carlizzi CN. Aspergillus infection of the L3-L4 disc space in an immunosuppressed heart transplant patient. Spine (Phila Pa 1976). 1990; 15 (12):1369. 1373. http://dx.doi.org/10.1097/00007632-199012000-00027.

[2] ErsoyA.DizdarOA.OrucKocA,AkalinH.et al. Aspergillus Fumigatus Spondylodiskitis in Renal Transplant Patient: Voriconazole Experience ExpClinTransplant. 2011 Aug; 9 (4):265-9.

[3] Gerlach UA, Kohler S, Sauer IM, et al. Aspergillus spondylodiscitis after multivisceral transplantation. Ann Transplant. 2009; 14 (4):52-57.

[4] Hummel M, Schüler S, Weber U, et al. Aspergillosis with Aspergillus osteomyelitis and diskitis after heart transplantation: surgical and medical management. J Heart Lung Transplant. 1993; 12 (4):599-603.

[5] Maschmeyer G, Haas A, Cornely OA. Invasive aspergillosis: epidemiology, diagnosis and management in immunocompromised patients. Drugs. 2007; 67 (11):1567-601. http://dx.doi.org/10.2165/00003495200767110-00004.

[6] Park SB, Kang MJ, Whang EA, Han SY, Kim HC. A case of fungal sepsis due to aspergillus spondydiscitis followed by cytomegalovirus infection in a renal transplant recipient. Transplant Proc. 2004; 36 (7):2154-2155. http://dx.doi.org/10.1016/j.transproceed.2004.08.049.

[7] Rüping MJ, Vehreschild JJ, Cornely OA. Patients at high risk of invasive fungal infections: when and how to treat. Drugs. 2008; 68 (14):1941-62. http://dx.doi.org/10.2165/00003495-200868140-00002.

[8] Salvalaggio PR, Bassetti M, Lorber MI, et al. Aspergillus vertebral osteomyelitis after simultaneous kidney-pancreas transplantation. Transpl Infect Dis. 2003; 5 (4):187-190. http://dx.doi.org/10.1111/j.1399-3062.2003.00030.x.

[9] Tang TJ, Janssen HL, Van der Vlies CH, et al. Aspergillus osteomyelitis after liver transplantation: conservative or surgical treatment? Eur J GastroenterolHepatol. 2000; $12 \quad$ (1):123-126. http://dx.doi.org/10.1097/00042737-200012010-00022.

[10] Wéclawiak H, Garrouste C, Kamar N, et al. Aspergillus fumigatusrelated spondylodiscitis in a heart transplant patient successfully treated with voriconazole. Transplant Proc. 2007; 39 (8):2627-2628. http://dx.doi.org/10.1016/j.transproceed.2007.08.014. 